\title{
Conformational and Reaction Dynamic Coupling in Histidine Kinases: Insights from Hybrid QM/MM Simulations
}

\author{
Federico A. Olivieri, ${ }^{\perp}$ Osvaldo Burastero, ${ }^{\perp}$ Salvador I. Drusin, Lucas A. Defelipe, Diana E. Wetzler, \\ Adrián Turjanski, and Marcelo Marti*
}

Cite This: https://dx.doi.org/10.1021/acs.jcim.9b00806

Read Online

ACCESS

Llll Metrics \& More

Article Recommendations

Supporting Information

ABSTRACT: Histidine kinases (HK) of bacterial two-component systems represent a hallmark of allosterism in proteins, being able to detect a signal through the sensor domain and transmit this information through the protein matrix to the kinase domain which, once active, autophosphorylates a specific histidine residue. Inactive-to-active transition results in a large conformational change that moves the kinase on top of the histidine. In the present work, we use several molecular simulation techniques (Molecular Dynamics, Hybrid $\mathrm{QM} / \mathrm{MM}$, and constant $\mathrm{pH}$ molecular dynamics) to study the activation and autophosphorylation reactions in L. plantarum WalK, a cis-acting $\mathrm{HK}$. In

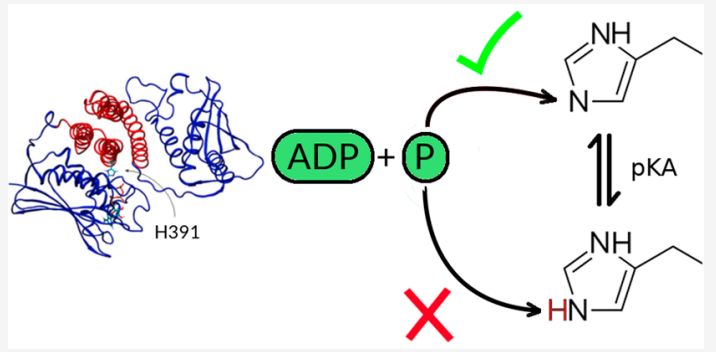
agreement with previous results, we show that the chemical step requires tight coupling with the conformational step in order to maintain the histidine phosphoacceptor in the correct tautomeric state, with a reactive $\delta$-nitrogen. During the conformational transition, the kinase domain is never released and walks along the HK helix axis, breaking and forming several conserved residue-based contacts. The phosphate transfer reaction is concerted in the transition state region and is catalyzed through the stabilization of the negative developing charge of transferring phosphate along the reaction.

\section{INTRODUCTION}

Two-component systems have been found to participate in the sensing of environmental stimuli in almost all bacterial species, as well as in plants and fungi. ${ }^{1,2}$ The first component, a histidine kinase-type sensor ( $\mathrm{HK})$, receives a stimulus, autophosphorylates the conserved phosphorylatable histidine (pHis) residue, and subsequently transfers the phosphate to an aspartic residue on the second component, called the response regulator, which generally acts as a transcription factor, regulating gene expression. ${ }^{3}$

Two-component systems are vital components of the bacterial ability to adapt to environmental changes. HKs have been found to sense many different signals including redox potential, gases, temperature, $\mathrm{pH}$, and light ${ }^{4-9}$ and are often important aspects of bacterial persistence and virulence. For example, the DosSTR system has been shown to be vital for $M$. tuberculosis infections ${ }^{10}$ and many different kinds of mutations in WalK $\mathrm{HK}$ of $S$. aureus confer vancomycin resistance. $^{11-13}$ This makes HKs attractive targets for drug design and therefore interesting study subjects.

HKs usually have a modular architecture with at least two distinct functional domains: a sensor domain and a conserved catalytic core. ${ }^{14}$ The sensor domains are highly variable, belonging to multiple families, such as PAS, GAF, LOV, and dCache among others. ${ }^{15,16}$ This variability allows the coupling of a wide range of stimulus to output responses through a conserved phosphorylation and transfer pathway. The kinase domain contains the dimerization and histidine phospho- acceptor (DHp) subdomain and the catalytic and ATP-binding (CA) subdomain. The DHp forms a coiled-coil that is the backbone of the $\mathrm{HK}$, connected at the $\mathrm{N}$-terminal end to the sensor domain and at the C-terminal to the CA. When the sensor domain detects the stimulus, a conformational change occurs that is transmitted along the coiled-coil backbone through the DHp, leading to a large motion of the CA toward the pHis and ultimately to autophosphorylation (Figure 1).

Their relevance and paradigmatic nature, as allosteric signal transduction proteins, promoted the structural studies of $\mathrm{HK}$, and several crystal structures of different $\mathrm{HK}$ domains in different states are available. ${ }^{17}$ The most common and easily accessible conformation shows the $\mathrm{DHp}$ in, what we refer to as, the inactive state. This conformation shows an overall symmetric dimeric structure in which the ATP from the CA is far (more than $17 \AA$ ) from the corresponding pHis (Figure 1A) (PDB ID $4 u 7 n$ ). Key to understanding these proteins, and to the present work, is the fact that for only four HKs an autokinase competent (which we call active) structure has been determined, namely, CpxA, EnvZ, Vick, and WalK (see Table S1 for the corresponding PDB IDs). In this conformation, the ATP gamma phosphate lies within the

Special Issue: Molecular Simulation in Latin America: Coming of Age

Received: September 18, 2019

Published: January 10, 2020 

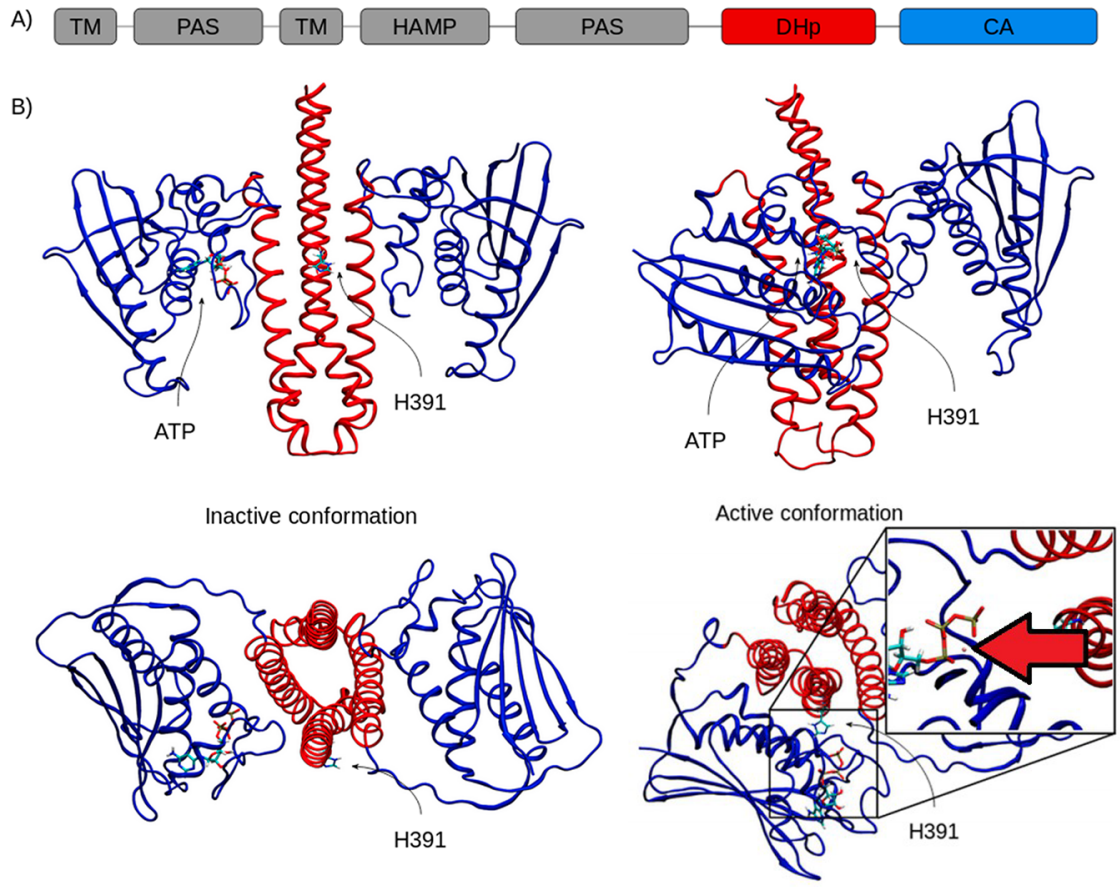

Figure 1. Domain structure of the Lactobacillus plantarum WalK (A) and its crystallographic structures of the active and inactive conformations (B). TM denotes transmembrane domains. This domain organization is characteristic of Bacillus WalK, while the Streptococcal homologues (VicK) lack the extracellular fragment and Mycobacterium homologues (MtrB) lack the cytoplasmic PAS domain. ${ }^{20}$ Inset shows the relative position of the ATP-P ${ }^{\gamma}$ and Phosphoacceptor His. The magnesium ion bound to the ATP is presented in pink.

reactive distance (ca. $4 \AA$ ) of the pHis (Figure 1B), allowing the study of the autophosphorylation reaction. However, further evidence of the actual catalytic capacity of these structures and the underlying mechanism of the chemical step is scarce. ${ }^{18,19}$ Also interesting is the fact that all these structures are asymmetric since only one of the CA-DHp pairs is found in the active conformation.

Another key detail about $\mathrm{HK}$ is that since they are dimers the CA can either phosphorylate the pHis from the same subunit (i.e., cis-autophosphorylation) or that from the other subunit (i.e., trans). Biochemically, both types of HKs have been shown to exist, and it is not clear if this issue has biological or evolutionary relevance. Structurally, structures of cis- and trans-active states are quite similar, showing the CA moving in the same direction, the difference resulting from alternative arrangements of the four-helix bundle of the $\mathrm{DHp}^{21}$ A comparison between both inactive and active conformations also highlights the necessary conformational changes required for $\mathrm{HK}$ activation, which comprise (i) intrinsic changes in the DHp coiled-coil and (ii) translation of one CA with respect to the DHp. However, little is known about the dynamics of the transition and the coupling between conformational and chemical steps.

In the last decades, molecular simulations have contributed significantly to our understanding of protein function, in many aspects, including protein dynamic conformational transitions as well as chemical catalysis. ${ }^{22}$ The field of protein dynamics is mostly studied using so-called classical (or force field)-based methods where bonds are modeled with strings, and interactions of nonbonded atoms are reduced to electrostatic (Coulomb) and dispersive (van der Waals) terms. They allow studying protein dynamics in the range from $100 \mathrm{~s}$ ns to $1 \mu \mathrm{s}$. On the other hand, to study catalysis and the underlying enzyme reaction mechanisms, classical approaches are unsuitable, since chemical reaction requires a quantum mechanics (QM) model, but they are computationally prohibitive for protein systems containing thousands of atoms. Multiscale simulations, such as QM/MM methods, combine the best of both worlds by only representing at a quantum scale the reactive part of the system, while the rest is treated with a classical force field. ${ }^{23,24}$ They have been widely used to study enzyme reactions including those related to phosphate chemistry. In particular, to study reaction mechanisms, QM/MM methods use some advanced reaction coordinate sample method, like steered molecular dynamics $^{25-27}$ or umbrella sampling, ${ }^{28}$ to accurately estimate the free energy along the reaction.

In previous work from our group, ${ }^{19}$ we used multiscale modeling techniques (coarse grain, $\mathrm{MD}$ and $\mathrm{QM} / \mathrm{MM}$ ) to study both HK conformational and chemical steps in CpxA, a trans-acting $\mathrm{HK}$ which senses envelope stress response by activating expression of downstream genes. Our previous results showed two important aspects that suggested there is tight coupling between CpxA conformational and chemical steps. First, we showed that the CA domain does not diffuse freely after release from the inactive to the active conformation, but instead, it "walks" along the DHp and is never released. The walking also fine tunes the tautomeric state of the pHis, allowing it to become phosphorylated and thus ultimately controlling the chemical step. Previous results also suggested that in a CpxA active state the most stable pHis tautomeric state corresponds to a doubly protonated state, which is therefore blocked to accept the phosphate.

In the present work, we explore the conformational activation and autophosphorylation mechanism of WalK, a cis-acting $\mathrm{HK}$ involved in the cell wall metabolism and cell growth in multiple species. ${ }^{29-32}$ Our results show that the 
Table 1. Constraints Used for All-Atom Molecular Dynamics

\begin{tabular}{lll}
\multicolumn{1}{c}{ Type } & \multicolumn{1}{c}{ Atoms } & \multicolumn{1}{c}{ NMR constants as defined in AMBER18 } \\
Distance & $\mathrm{N}_{\text {HIS }}^{\varepsilon}-\mathrm{P}^{\gamma}{ }_{\text {ATP }}$ & $\mathrm{r} 1=0.0, \mathrm{r} 2=1.0, \mathrm{r} 3=4, \mathrm{r} 4=14, \mathrm{rk} 2=60, \mathrm{rk} 3=60$ \\
Angle & $\mathrm{O}^{3 \mathrm{~B}}{ }_{\mathrm{ATP}}-\mathrm{P}_{\mathrm{ATP}}^{\gamma}-\mathrm{N}_{\mathrm{HIS}}^{\varepsilon}$ & $\mathrm{r} 1=110.0, \mathrm{r} 2=170, \mathrm{r} 3=190, \mathrm{r} 4=250.0, \mathrm{rk} 2=60, \mathrm{rk} 3=60$ \\
Distance & $\mathrm{O}^{\mathrm{E2}}{ }_{\mathrm{GLU}}-\mathrm{H}_{\mathrm{HIS}}^{\delta}$ & $\mathrm{r} 1=0.0, \mathrm{r} 2=1.0, \mathrm{r} 3=2.2, \mathrm{r} 4=14, \mathrm{rk} 2=60, \mathrm{rk} 3=60$
\end{tabular}

chemical and conformational steps are tightly coupled and highlight interesting mechanistic aspects of both processes.

\section{METHODS}

Starting Structures. All the structures of histidine kinases were taken from the PDB database. Autokinase-competent (active) and -inactive conformations of WalK were taken from crystal structures $4 \mathrm{U} 70$ and $4 \mathrm{U} 7 \mathrm{~N}$, respectively. Two other histidine kinases in active conformations, VicK and a chimeric EnvZ that incorporates the DHp loop of HK853 (EnvZ $\left.Z_{\text {chim }}\right)$, were taken from crystal structures $4 \mathrm{I} 5 \mathrm{~S}$ and $4 \mathrm{KP} 4$, respectively. ${ }^{21,33}$ The structures of active WalK and $\mathrm{Envz}_{\text {chim }}$ had ATP analogs (AN2 and ANP, respectively) that were replaced with ATP molecules, while one was added to the structure of VicK which had no ligand at all. Missing loops were completed, and the initial helix of WalK was extended using Modeler. ${ }^{34}$

Residue Conservation Analysis. A PSI-BLAST with default parameters was performed using the Lactobacillus plantarum WalK sequence as a query. After five iterations, those sequences with over $70 \%$ positives were selected and used to build an HMM using the HMMER package. ${ }^{35}$ Residue conservation was taken as the probability of the match state for a particular residue.

All-Atom Molecular Dynamics Simulation Parameters. All-atom molecular dynamics (MD) simulations were performed using the PMEMD module of the AMBER $18^{36}$ package with the FF14SB force field ${ }^{37}$ in explicit TIP3P ${ }^{38}$ solvent. All simulations were performed at $300 \mathrm{~K}$ and constant pressure using the berendsen barostat. Parameters for ATP were taken from the literature. ${ }^{39}$

Steered Molecular Dynamics. To study the transition between the two conformational states of WalK, we used steered molecular dynamics (sMD), using two RMSDs simultaneously as the reaction coordinate using as references the inactive and active structures, respectively.

The region considered for the RMSDs consisted of the alpha carbons of the DhP domain of both protomers and the nonloop regions of the active protomer's CA domain. Here, 10 ns of equilibration $\mathrm{MD}$ was performed before beginning the $\mathrm{sMD}$, and then, the sMD simulations were run over $110 \mathrm{~ns}$ at a 2 fs time step, resulting in a pulling speed of $0.07 \AA / \mathrm{s}$. The force constant used was $430 \mathrm{kcal} / \mathrm{mol} \cdot \AA^{2}$.

The RMSD against the active reference structure varies from $\sim 1.6 \AA$ for equilibrium simulations of the active state to $\sim 8.4 \AA$ for equilibrium simulations of the inactive state. Similarly, RMSD against the inactive reference structure varies from $\sim 1.6$ $\AA$ for equilibrium simulations of the inactive state to $\sim 8 \AA$ for equilibrium simulations of the active state. When plotting values from sMD simulations, the difference between RMSDs was used as the reaction coordinate, which thus ranges from approximately -6.8 to $6.4 \AA$.

It is important to remark that using using a double RMSD as the $\mathrm{RC}$ tends to reduce possible entropic bias in the free energy profile (FEP) end states with respect to single RMSD
$\mathrm{RC}$, avoiding restricting the conformational space available when one of the two RMSDs is high.

Umbrella Sampling (US) Simulations. Intermediate snapshots of the sMD transition were taken to perform US simulations and obtain the corresponding FEP. In total, 49 windows of 14 ns US simulations were done restraining the double reaction coordinate defined in the sMD with a force constant of $100 \mathrm{kcal} / \mathrm{mol} \cdot \AA^{2}$. The atom selection was the same as the one used in the sMD. The first two nanoseconds were discarded as equilibration phase, and the remaining 12 ns were used for the construction of the FEP as implemented in WHAM. $^{40}$

Briefly, to make the FEP in Figure 3, we used the following protocol. First, we constructed the 2D FEP using the double RMSD described above. Then, we defined a new variable as the RMSD of the inactive state minus the RMSD of the active state and plotted this new variable versus the corresponding energy value. To make the inset in Figure 3, we constructed a 1D FEP by using only the values of the RMSD to the active state, which highlights the region close to the active structure. In this case, statistical uncertainty was calculated by a block bootstrap as implemented in WHAM (50 bootstrap samples). Correlation time of each window measurement was taken into account by estimating it as the lag time where the autocorrelation was similar to white noise (95\% confidence interval). In both cases, US windows having a correlation time larger than 2 ns were removed from the analysis.

The FEPs of the pHis in the HIP (doubly protonated) state or the $\mathrm{R} 434 \mathrm{~L}$ mutant were constructed with the same methodology. In this case, we rerun in the new states only the US windows with RMSD to the active conformation less than $4 \AA$. For the remaining part of the reaction coordinate, the US windows of the HID ( $\delta$-protonated) state were used. Histogram overlaps of the US windows for the RMSD to the active state are shown in Figure S1.

Constant pH Molecular Dynamics. With the aim of investigating the protonation state of the $\mathrm{pHis}$ along the conformational change, all-atom constant $\mathrm{pH}$ molecular dynamics (CpHMD) simulations were performed on the active and inactive structures of $\mathrm{WalK}, \mathrm{EnvZ}_{\mathrm{chim}}$, and $\mathrm{VicK}$, as well as 11 transition structures of WalK. In this method, developed by Mongan et al. $^{41}$ and previously used in our group, ${ }^{19}$ the titratable residue changes its protonation and tautomeric state according to its electrostatic environment and the $\mathrm{pH}$ at which the simulation is set. The possible tautomeric states are HIP, HID, and HIE ( $\varepsilon$-protonated). CpHMD simulations of $50 \mathrm{~ns}$ at $\mathrm{pHs}$ from 2 to 12 with the backbone harmonically restrained (with a force constant of $0.1 \mathrm{kcal} / \mathrm{mol}$. $A^{2}$ ) were carried out for all the mentioned structures. Subsequently, the fractions of the simulation in different protonation states at each $\mathrm{pH}$ were extracted, and the residue's $\mathrm{pK}_{\mathrm{a}}$ was calculated by fitting the Henderson-Hasselbalch equation to the curve of the protonated fraction vs $\mathrm{pH}$.

Reaction Free Energies Using Hybrid QM(DFT-GGA)/ MM Simulations. In order to obtain the free energy profile for the histidine autophosphorylation reaction, we performed 
sMD combined with a QM/MM scheme. The QM system was treated with a GPU implementation of a density functional theory method with the generalized gradient approximation (DFT GGA) $^{42}$ and the MM system with the Amber FF14SB force field. ${ }^{37}$ The $\mathrm{QM} / \mathrm{MM}$ implementation is freely available. $^{43}$

First, to get an adequate reactant state, five $50 \mathrm{~ns}$ replicas of all-atom molecular dynamics at $300 \mathrm{~K}$ and density fixed to 1 with some restraints were done (Table 1). For each one, snapshots every $10 \mathrm{~ns}$ were taken resulting in 25 snapshots. Ten of them were selected by visual inspection and cooled to $10 \mathrm{~K}$. Subsequently, the QM/MM regime was turned on, and the system energy was minimized using 1000 steps of gradient descent. The QM atoms calculations were performed with the $\mathrm{PBE}^{44}$ functional using the DZVP basis, except for magnesium for which we used a 6-31G basis. Covalent boundaries were treated with the AMBER link atom method.

The previous step was done to ensure appropriate bond distances for the QM system (which may differ from the ones presented in the amber ff14SB force field) and avoid the nonconvergence of the DFT (density functional theory) method. Then, one minimized structure was selected for the following $\mathrm{QM} / \mathrm{MM}$ dynamics protocol: Temperature was raised from 10 to $300 \mathrm{~K}$ in 6.5 ps keeping the volume constant. Density was adjusted to 1 by performing a 6 ps dynamic with the pressure constant at $1 \mathrm{~atm}$ and the temperature at $300 \mathrm{~K}$. Finally, the reaction coordinate defined as the cleaved bond distance $\left(\mathrm{P}_{\mathrm{ATP}}^{\gamma}-\mathrm{O}^{3 \mathrm{~B}}{ }_{\mathrm{ATP}}\right)$ minus the formed bond distance $\left(\mathrm{P}_{\mathrm{ATP}}^{\gamma}-\mathrm{N}_{\text {HIS }}^{\varepsilon}\right)$ was fixed to $-2.4 \AA$, and 18 equilibrated snapshots of the reactant state were taken at 2 ps intervals. Along the whole protocol, the alpha carbons of the protein residues were restrained with a quadratic positional restraint weight of $5 \mathrm{kcal} / \mathrm{mol}$, and a time step of $1 \mathrm{fs}$ was used.

The 18 starting reactant structures were used as starting points for the sMDs. The reaction coordinate was taken from -2.4 to $2.4 \AA$ using a pulling speed of $0.23 \AA / \mathrm{ps}$, and the Jarzynski estimator was used to compute the FEP. ${ }^{45}$ For the pulling trajectories, a time step of 0.5 fs was used with the intention of allowing hydrogen transferences. Individual works of the sMD can be observed in Figure S2. A detailed explanation of the FEP determination along with the error estimation are present in the Supporting Information, Free energy profile (FEP) estimation of the autophosphorylation reaction section.

\section{RESULTS}

Dynamics of Inactive-to-Active HK Conformational Transition. To analyze in atomic detail the WalK inactive-toactive conformational transition, we first performed targeted steered $\mathrm{MD}$, using as RC the double RMSD with respect to both reference structures as detailed in the Methods section.

From a global perspective, the first important point to notice is that the CA domain does not detach from DHp and rebinds in an active conformation but instead walks along the DHp. This result is in agreement with our results obtained for CpxA and highlights the relevance of the gradual formation of contacts between DHp and CA along the transition, as described below for WalK. The first important contact to form during the change is the Arg434-Asp502 interaction, for which it is necessary to break the Arg434-Asn510 hydrogen bond present in the inactive state. This brings the CA into a position where Arg469 is able to form a bond with Glu428. Finally, Asn510 interacts with Glu392. The formation of this interaction pulls the pHis and His391 into the catalytic pocket through its interaction with Glu392 (Table 2). Interestingly,

Table 2. Important Contacts between DHp and CA Domains of WalK and Their Homologues in $\mathrm{CpxA}^{a}$

\begin{tabular}{cc} 
WalK & CpxA \\
\hline Glu392(0.407)-Asn510(0.367) & Glu249-Asn356 \\
Arg434(0.430)-Asp502(0.453) & Ser286-Asn348 \\
Arg434 $(0.430)-A s n 510(0.367)$ & Ser286-Asn356 \\
Glu428 $(0.407)-$ Arg469(0.430) & Glu280-Asn320
\end{tabular}

${ }^{a}$ Amino acids in bold belong to the DHp domain and amino acids in italic to the CA domain. The numbers between parentheses represent the conservation score of the corresponding residue among WalK homologues. The conservation score was taken as the probability of the most probable match state in an HMM built from a selection of WalK homologues with identities of $70 \%$ with respect to the $L$. plantarum sequence. The average conservation score for nonconserved residues was 0.1 .

these residues are highly conserved among WalK homologues. Furthermore, even among more distant HKs like WalK and CpxA (Table 2), the capability to form hydrogen bonds is conserved in residues in the corresponding positions of the DHp and CA. These residues are shown in Figure 2.

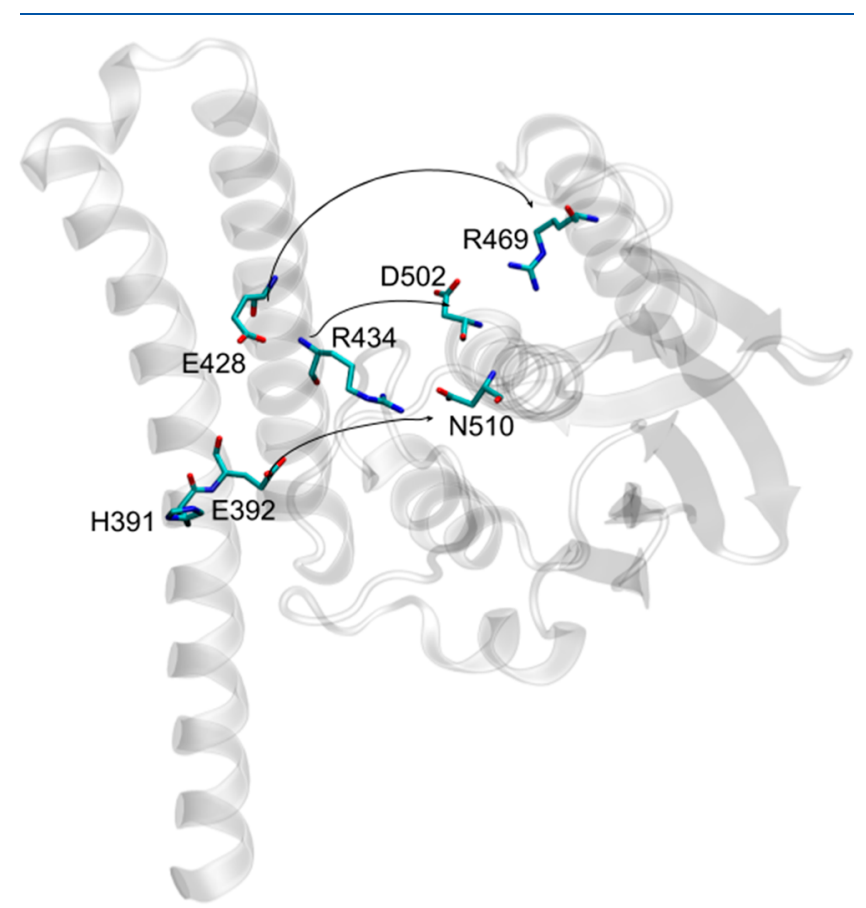

Figure 2. Inactive structure of WalK showing the contacts that form during the activation conformational transition.

It is interesting to note that, as the CA domain approaches the reactive histidine, the latter tends to move away from the catalytic pocket. This evidence, together with the $\mathrm{p} K_{\mathrm{a}}$ calculations, further supports the hypothesis that the activation and phosphorylation reactions must be concerted.

Umbrella Sampling Simulations of Inactive-to-Active Transition. To gain a thermodynamic viewpoint of the conformational transition, we extracted intermediate structures from the inactive-to-active sMD trajectory and used them as starting points to determine the corresponding free energy profile (FEP) using umbrella sampling (US). Briefly, the FEP 
profile was built from 49 windows (each 14 ns long MD) using the same RC. We determined the FEP for wt (wild-type) protein with pHis in the HID state (Figure 3) and also

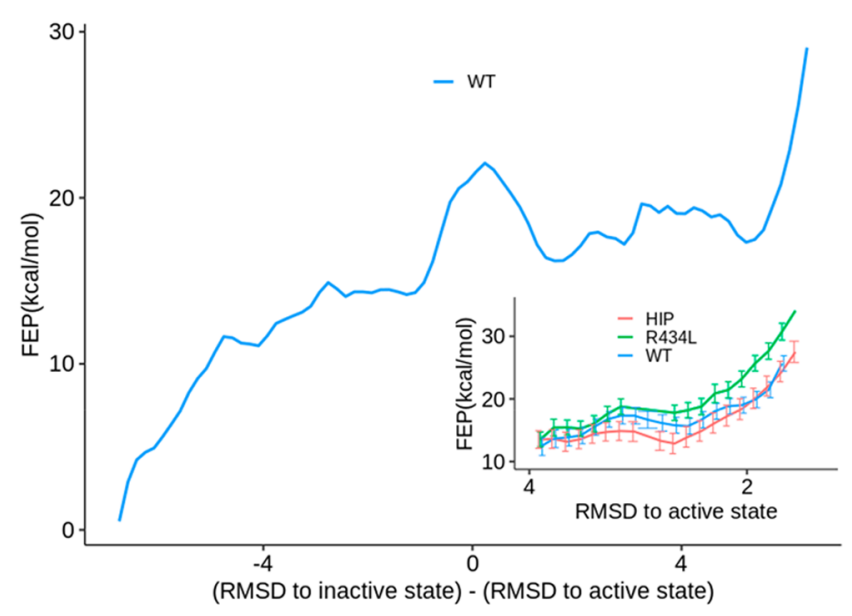

Figure 3. Free energy profile versus $\triangle \mathrm{RMSD}$ derived from the US simulations of the WT WalK in the HID state. The reaction coordinate for the simulations was defined as the double RMSD to the active state and inactive state as defined in the Methods section. Inset: FEP vs RMSD to the active state plot for the WT WalK in HID state (blue), HIP state (red), and R434L mutant (green).

analyzed the change of the last part of the FEP by changing the pHis to the HIP state or by mutating R434 to leucine in some US windows close to the active conformation (RMSD < 4). As a result, we obtained a free energy profile of the active to inactive transition that can be qualitatively separated in three stages (Figure 3 inset). First, the energy raises about $10 \mathrm{kcal} /$ mol from RMSD $\sim 8$ to RMSD $\sim 7$, possibly due to initial breaking of CA-Dhp interactions. In the second stage, the energy remains stable until RMSD $\sim 5$. In this stage, CA-DHp interactions are formed and broken simultaneously in a walking like motion of the CA along the DHp. Finally, there is a small barrier that leads to a minimum at RMSD $\sim 2$, corresponding to phosphate transfer "active" state. Not surprisingly, when the conserved arginine 434 is mutated to leucine, the energy of the transition is greatly increased, and the minimum at RMSD $\sim 2$ almost disappears. On the other hand, when the pHis is in the HIP state, the "active" conformation is more stable. This result suggests, that as previously observed for $\mathrm{CpxA}$, in the active conformation, the doubly protonated His predominates.

It is important to mention that due to the large magnitude of the observed conformational change and underlying difficulty to adequately sample all possible orthogonal conformations required to converge the FEP along the selected RC, the presented FEP reveals more about the differences between WT HID, HIP, and R434L than about its intrinsic free energy values which is possibly overestimated and should be taken with care.

CA Release as an Alternative Path toward Active State. As a possible alternative activation mechanism, we analyzed a CA "release and rebind" scenario. We computed the corresponding FEP in two steps using a target MD. First, we computed the free energy required to detach the CA from the DHp. The results show that to release the CA almost $40 \mathrm{kcal} /$ mol are required (Figure S3). This is not unexpected since several tight contacts have to be broken, R434-N510 being the last and most resistant. The second step (Figure S3), comprising the rebinding of the $\mathrm{CA}$ in an active position, on the contrary, shows a clear free energy gain (of ca. $30 \mathrm{kcal} /$ $\mathrm{mol}$ ), justified by the concomitant formation of several tight contacts. Summarizing, it is clear that a "release and rebind" mechanism implies a much higher barrier compared to the "walking" mechanism. The origin of the difference possibly lies in the fact that during the walk contacts are broken and reformed in an equilibrated stepwise manner, avoiding paying the energetic cost of breaking the whole CA-DHp interaction.

Phosphoacceptor Histidine $\mathrm{p} K_{\mathrm{a}}$ is Conformation Dependent and Becomes "Blocked" in the Active State. To analyze how the pHis tautomeric state (and $\mathrm{p} K_{\mathrm{a}}$ ) is related to the HK conformational state, and complement our previous results on trans-acting CpxA, we performed $\mathrm{CpH} \mathrm{MD}$ in all available $\mathrm{HK}$ active structures. They correspond to WalK, VicK (a WalK analog in Streptococcus mutans), and chimeric EnvZ kinase, which is involved in the regulation of osmolarity but has had its DHp loop replaced with the DHp loop from
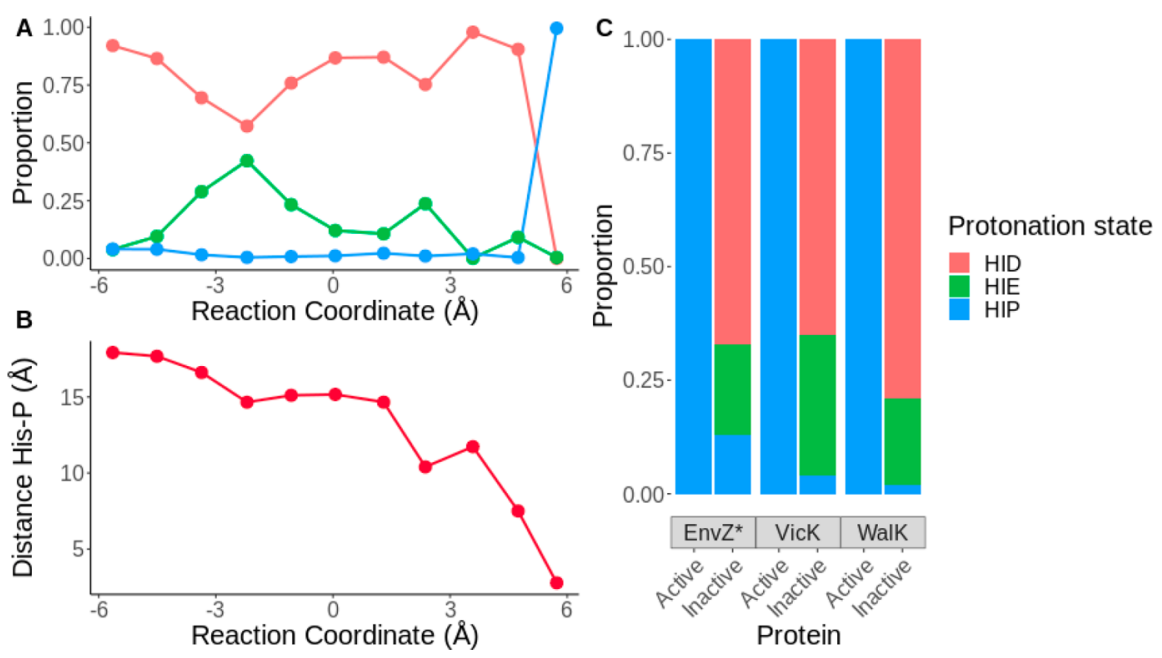

Figure 4. Proportion of each histidine tautomer (A) and histidine-phosphate distance (B) along the inactive-to-active transition of WalK. Proportion of tautomers at $\mathrm{pH} 7$ for the EnvZ $\mathrm{Chim}_{\mathrm{C}}$, VicK, and WalK. (C). Panels A and B are plotted using the conformational RC (difference between both reference structures' RMSDs as described in the Methods section) as the $X$-axis. 


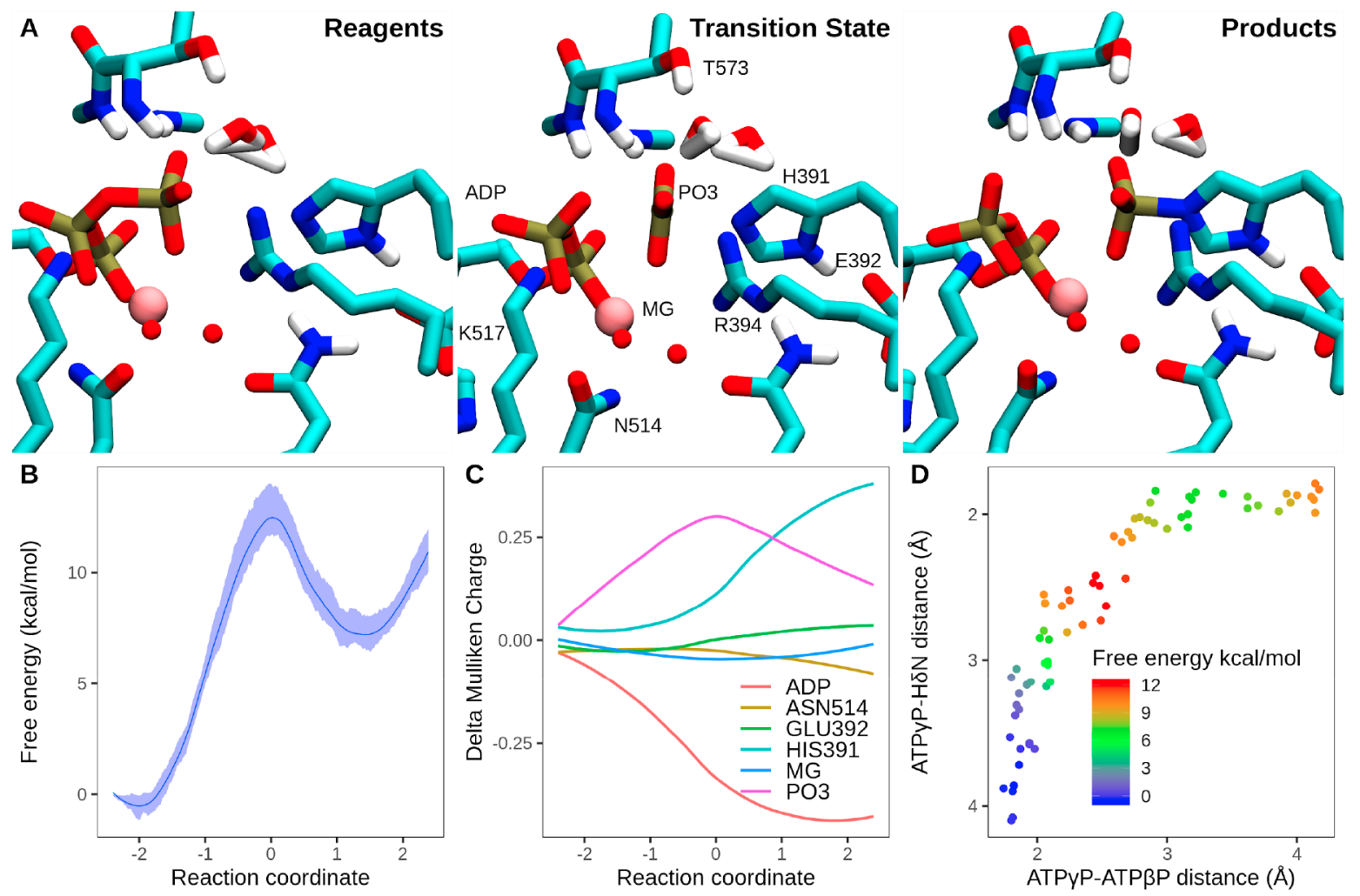

Figure 5. QM/MM results. (A) Atomistic representation of the reactive (R), transition (TS), and product states (P). (B) Free energy profile of the histidine kinase autophosphorylation reaction in WalK. (C) Change in Mulliken charges along the reaction coordinate. The selected moieties were the side chains from His391 and Glu392, the magnesium ion, ADP, and the phosphoryl group. (D) Corresponding MOFJ plot of the histidine kinase autophosphorylation reaction using bond forming and bond breaking distance from one representative sMD. The reaction coordinate in panels $\mathrm{B}$ and $\mathrm{C}$ corresponds to the difference between the $\mathrm{P}_{\text {ATP }}^{\gamma}-\mathrm{O}^{3 \mathrm{~B}}{ }_{\text {ATP }}$ bond and the $\mathrm{P}_{\text {ATP }}^{\gamma}-\mathrm{N}^{\varepsilon}{ }_{\text {HIS }}$ bond.

the HK853 kinase. The results show that in the inactive state HIS391 is in the HID tautomer. This tautomer is stable all throughout the transition, but as the Histidine residue slides into the catalytic pocket, it switches to a HIP state (Figure 4A and $\mathrm{B}$ ). This result was consistent for all three proteins analyzed (Figure 4C).

Visual inspection of the corresponding structures allows rationalizing this observation. In the active conformation, the $\mathrm{P}_{\text {ATP }}^{\gamma}$ points directly toward the pHis epsilon nitrogen; therefore, when protonated, a very strong salt bridge is established that strongly favors a positive His. Moreover, when pHis is forced to adopt a HID state, the active complex becomes unstable, and pHis moves away from the reactive conformation into a conformation unable to accept the phosphate. The $\delta$-nitrogen of the pHis is also protonated, since it is hydrogen bonded to the conserved adjacent acid residue. This interaction which is also present in the inactive state is the main interaction responsible for HID predominance in this case. It is important to point out that the crystallographic structures available in the active state were obtained with a nonhydrolyzable ATP analog or correspond to an apo structure (i.e., without ATP). In these particular structures, the calculation of $\mathrm{p} K_{\mathrm{a}}$ may not be biologically relevant as we are forcing the system to establish an interaction with the gamma phosphate of the ATP analog.

We also determined the pHis tautomeric state preference along the whole conformational transition to try to determine when and why the $\mathrm{p} K_{\mathrm{a}}$ changes. The results, presented in Figure 4A, show that the pHis remains primarily in the HID state all throughout the conformational change, only switching to HIP once the protein is fully in its active conformation, with the pHIS epsilon nitrogen less than $4 \AA$ from the $\mathrm{P}_{\text {ATP }}^{\gamma}$ (Figure 4B).

Autophosphorylation Reaction Mechanism. To complete the picture of $\mathrm{HK}$ activation mechanism, we analyzed the chemical step, i.e., the autophosphorylation reaction, using hybrid QM/MM steered molecular dynamics at the DFT(PBE/DZVP) level and determined the associated free energy profile (FEP). ${ }^{42,45}$ The selected QM/MM region was based on our experience with kinase reactions, ${ }^{19,46}$ including previous work on CpxA, and comprised the side chain of pHis (His391 in this case), the side chain of adjacent Glu392, part of the ATP (from the $\mathrm{C5}^{\prime}$ atom to the $\gamma$-phosphate), a $\mathrm{Mg}^{2+}$ ion, two waters, and the side chain of Asn514 implicated in $\mathrm{Mg}^{2+}$ coordination. The chosen reaction coordinate was the combination of the cleaved bond distance $\left(\mathrm{P}_{\text {ATP }}^{\gamma}-\mathrm{O}^{3 \mathrm{~B}}{ }_{\text {ATP }}\right)$ minus the formed bond distance $\left(\mathrm{P}_{\text {ATP }}^{\gamma}-\mathrm{N}^{\varepsilon}{ }_{\text {HIS }}\right)$, leaving the $\mathrm{H}^{\delta}{ }_{\text {HIS }}$ to freely move. More details about the methods to obtain the FEP can be found in the Methods section.

The obtained results are presented in Figure 5. The catalytic pocket of WalK is shown in Figure 5A. The FEP shows a barrier of ca. $12 \mathrm{kcal} / \mathrm{mol}$ (Figure $5 \mathrm{~B}$ ), which is slightly smaller than the previously reported barrier for the same reaction in the trans-histidine kinase CpxA (Table 2). In both cases, the reaction is slightly endergonic (ca. $5 \mathrm{kcal} / \mathrm{mol}$ ). Analyses of the electronic and dynamical parameters along the reaction (see Mulliken charges and MOJF plots in Figure 5C and D) show that the reaction is mainly associative in the early steps; 
Table 3. Summary of Chemical Step “Autophosphorylation” Results

\begin{tabular}{|c|c|c|c|c|c|}
\hline Histidine tautomer & Parameter & Value $(\mathrm{kcal} / \mathrm{mol})$ & Environment & Comments & ref \\
\hline HID & $\Delta G^{\#}$ & 25 & Water & & 23 \\
\hline HID & $\Delta G^{\#}$ & 12 & WalK HK & & This work \\
\hline HID & $\Delta G^{\#}$ & 16 & CpxA HK & & 23 \\
\hline HIP & $\Delta G^{\#}$ & 25 & СрхА HK & pHis donates first its proton to the ATP. & 23 \\
\hline HIP & $\Delta E^{\#}$ & 25 & CheA HK & pHis donates first its proton to the ATP. & 22 \\
\hline
\end{tabular}

initially, the $\mathrm{N}_{\text {HIS }}^{\delta}$ and $\mathrm{P}_{\text {ATP }}^{\gamma}$ are brought close together and concerted in the transition state (TS) region. The TS is symmetric with a trigonal bipyramidal phosphate equally distant from $\mathrm{N}_{\text {HIS }}^{\delta}$ and $\mathrm{P}_{\text {ATP. When compared to the }}^{\gamma}$ uncatalyzed reaction in water $\left(\Delta G^{\#}=25 \mathrm{kcal} / \mathrm{mol}\right.$ and $\Delta G^{0}$ $=18 \mathrm{kcal} / \mathrm{mol}$ ), the catalytic effect is evidenced as well as its underlying nature (Table 2). Key to catalysis is to screen the charge that is developed in transferring phosphate along the TS (transition state). In this case, the TS is directly stabilized by several conserved hydrogen bonds and charge interactions (Figure 5A).

Moreover, along the phosphoryl transfer reaction, the distance of the hydrogen $\mathrm{H}_{\text {HIS }}^{\delta}$ to the oxygen OE1 from the adjacent glutamic tends to diminish, and even in two sMD, a complete proton transfer was observed (data not shown). Therefore, as suggested by Marina et al., ${ }^{47}$ Glu392 seems to have a role not only in conferring the phosphorylatable histidine preference for the HID tautomeric state but also acts as a general base to capture the $\mathrm{H}_{\text {HIS }}^{\delta}$. A considerable change of the mulliken charges from the histidine moiety can also be noticed in Figure 5C. This charge is "acquired" by the ADP moiety that ends more negatively charged.

\section{DISCUSSION}

Understanding the underlying mechanistic details of the HK activation mechanism is a very active research area, and several questions remain unanswered. Particularly relevant, and the focus of the present work, is the relationship between the inactive-to-active conformational transition (conformational step) triggered by stimuli detected the sensor domain and the phosphorylation reaction (the chemical step). In a previous work from our group on trans-acting CpxA HK, we showed that while in the inactive conformation the pHis is predominantly in the HID tautomer and thus ready to receive the phosphate, once the active state is fully achieved, strong interactions of pHis with negatively charged ATP result in a predominantly positively charged pHis (HIP state) which is thus unable to accept the phosphate. In the present work, we analyzed three new $\mathrm{HK}, \mathrm{EnvZ}_{\text {Chimera }}$ VicK, and WalK (a cisacting HK), for which an active state structure is available, confirming that in all cases $\mathrm{pHis}$ is predominantly protonated and blocked once interacting with the ATP. It is important to note that the experimental observation of a fully stable activelike state can only be achieved using nonhydrolyzable ATP analogs, such as AMPNP. For those structures obtained in the latter (Table S1), it is clear that a strong interaction exists between pHis and ATP-P $\gamma$ which is only possible when $\mathrm{pHis}$ is fully protonated.

In this context, we propose that autophosphorylation is achieved through a tight coupling between conformation and chemical steps that maintains the pHIS in a reactive tautomer (HID) until it comes close to the ATP, which would naturally lead to autophosphorylation, preventing reaching the HIP blocked state. Our results provide strong evidence supporting this hypothesis. First, as shown in Figure 4A for WalK, and as already observed in our group for CpxA, along the conformational transition, $\mathrm{pHis}$ remains predominantly in the HID state, until the very last, when the $\mathrm{P}_{\text {ATP }}^{\gamma}$ is less than $4 \AA$ from the pHis $-\mathrm{N} \delta$, and thus, the phosphate transfer reaction can begin. This makes it unnecessary for the pHis to deprotonate before phosphorylation, lowering the overall energy barrier of the reaction. Further support comes from the FEP of the conformational transitions, which shows that after initial breaking of $\mathrm{DHp}-\mathrm{CA}$ domain interactions, the CA walks through a free energy plane along the $\mathrm{DHp}$ until reaching the reactive conformation. Having a protonated $\mathrm{pHis}$ only alters the FEP in the final segment of the RC, contributing as expected favorably to the FEP, yielding a minimum where the protonated pHis interacts tightly with ATP, which corresponds to the structures observed experimentally.

Further support to the coupling hypothesis comes from an analysis of proton exchange rates, as reported by Liepinsh and Otting, ${ }^{48}$ which in the fastest limit approach a rate of once every $100 \mu \mathrm{s}$. In this context, assuming that conformational change occurs in the nano to few microsecond time scale, with the chemical step being even faster, leaves no time, even if active conformation with a high $\mathrm{p} K_{\mathrm{a}}$ is fully reached, to equilibrate the $\mathrm{pHis}$ side chain with the local $\mathrm{pH}$.

Concerning the conformational transitions, the main observation of the present work, which is inline with previous work for CpxA, is related to the fact that CA movement is best described as a walking of the CA along the DHp. This walking mechanism is directly related to the fact that both cis (WalK)and trans (CpxA)-acting HK show a similar pattern of contacts (many of which show high residue conservation) that break and form during the transition leading to a topologically similar active conformation, where the cis/trans preference is dictated solely by the conection of the a1 and a 2 helices of the DHp dimer that form the 4-helix bundle, as proposed by Ashenberg et al. ${ }^{49}$ Using sMD simulations, we explored an alternative mechanism where the CA detaches and rebinds to the DHp in the active conformation. This mechanism showed a significantly higher energy barrier than the walking mechanism due to the need to break several contacts in order to fully release the CA, while the walking mechanism forms new contacts as the inactive state contacts are broken.

Finally, looking at the chemical step, the following points stand out. The calculated $\Delta G^{\#}$ for the transition was $\sim 12 \mathrm{kcal} /$ mol, a value which is consistent with previous works exploring the HK family (Table 2). It is also interesting to note that the obtained barrier is significantly smaller than those observed for the alternative mechanism previously determined for both CheA and CpxA starting from a protonated pHIS that involves two steps: (1) proton transfer from the $\mathrm{N}^{\delta}{ }_{\text {HIS }}$ to the $\mathrm{P}_{\text {ATP }}^{\gamma}$ and subsequent small adjustment of both reactants (which requires 4 to $8 \mathrm{kcal} / \mathrm{mol}$ ) and (2) a transfer of a PO3H to the $\mathrm{N}^{\delta}$ HIS, displaying a barrier of more than $25 \mathrm{kcal} / \mathrm{mol}$ (similar or even higher than the uncatalyzed reaction) (Table 3). Clearly, these 
results also support our hypothesis that conformation and chemical steps must be coupled in order to maintain the pHIS as a tautomer capable of accepting the incoming phosphate.

\section{CONCLUSION}

In the present work we studied using molecular simulations, the conformational and chemical steps of histidine kinases. Our results show that the chemical step requires a tight coupling with the conformational step in order to maintain the histidine phosphoacceptor in the correct tautomeric state, with a reactive $\delta$-nitrogen. The reaction is concerted in the TS region which is stabilized by the conserved hydrogen and charged interactions with the transferring phosphate.

\section{ASSOCIATED CONTENT}

\section{SI Supporting Information}

The Supporting Information is available free of charge at https://pubs.acs.org/doi/10.1021/acs.jcim.9b00806.

Information as mentioned in the text. (PDF)

\section{AUTHOR INFORMATION}

\section{Corresponding Author}

Marcelo Marti - Departamento de Química Biologica, Facultad de Ciencias Exactas y Naturales, Universidad de Buenos Aires, Ciudad Universitaria, C1428EGA Ciudad Autónoma de Buenos Aires, Argentina; Instituto de Química Biológica de la Facultad de Ciencias Exactas y Naturales (IQUIBICEN) CONICET, Ciudad Universitaria, C1428EGA Ciudad Autónoma de Buenos Aires, Argentina; (1) orcid.org/00000002-7911-9340; Email: marti.marcelo@gmail.com

\section{Authors}

Federico A. Olivieri - Departamento de Química Biológica, Facultad de Ciencias Exactas y Naturales, Universidad de Buenos Aires, Ciudad Universitaria, C1428EGA Ciudad Autónoma de Buenos Aires, Argentina; Instituto de Química Biológica de la Facultad de Ciencias Exactas y Naturales (IQUIBICEN) CONICET, Ciudad Universitaria, C1428EGA Ciudad Autonoma de Buenos Aires, Argentina; (C) orcid.org/ 0000-0002-6253-453X

Osvaldo Burastero - Departamento de Química Biologica, Facultad de Ciencias Exactas y Naturales, Universidad de Buenos Aires, Ciudad Universitaria, C1428EGA Ciudad Autónoma de Buenos Aires, Argentina; Instituto de Química Biologica de la Facultad de Ciencias Exactas y Naturales (IQUIBICEN) CONICET, Ciudad Universitaria, C1428EGA Ciudad Autónoma de Buenos Aires, Argentina; 이이. orcid.org/ 0000-0003-4089-0434

Salvador I. Drusin - Departamento de Química Biológica, Facultad de Ciencias Exactas y Naturales, Universidad de Buenos Aires, Ciudad Universitaria, C1428EGA Ciudad Autónoma de Buenos Aires, Argentina; Instituto de Química Biológica de la Facultad de Ciencias Exactas y Naturales (IQUIBICEN) CONICET, Ciudad Universitaria, C1428EGA Ciudad Autonoma de Buenos Aires, Argentina; Área Física, Departamento de Químico-Física, Facultad de Ciencias Bioquímicas y Farmaćeuticas, Universidad Nacional de Rosario, S2002LRK Rosario, Santa Fe, Argentina; 이이.org/00000001-5350-514X

Lucas A. Defelipe - Departamento de Química Biológica, Facultad de Ciencias Exactas y Naturales, Universidad de Buenos Aires, Ciudad Universitaria, C1428EGA Ciudad
Autónoma de Buenos Aires, Argentina; Instituto de Química Biologica de la Facultad de Ciencias Exactas y Naturales (IQUIBICEN) CONICET, Ciudad Universitaria, C1428EGA Ciudad Autónoma de Buenos Aires, Argentina; European Molecular Biology Laboratory Hamburg, D-22607 Hamburg,

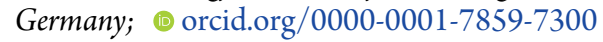

Diana E. Wetzler - Departamento de Química Biologica, Facultad de Ciencias Exactas y Naturales, Universidad de Buenos Aires, Ciudad Universitaria, C1428EGA Ciudad Autonoma de Buenos Aires, Argentina; Instituto de Química Biológica de la Facultad de Ciencias Exactas y Naturales (IQUIBICEN) CONICET, Ciudad Universitaria, C1428EGA Ciudad Autonoma de Buenos Aires, Argentina

Adrián Turjanski - Departamento de Química Biológica, Facultad de Ciencias Exactas y Naturales, Universidad de Buenos Aires, Ciudad Universitaria, C1428EGA Ciudad Autonoma de Buenos Aires, Argentina; Instituto de Química Biologica de la Facultad de Ciencias Exactas y Naturales (IQUIBICEN) CONICET, Ciudad Universitaria, C1428EGA Ciudad Autónoma de Buenos Aires, Argentina

Complete contact information is available at: https://pubs.acs.org/10.1021/acs.jcim.9b00806

\section{Author Contributions}

${ }^{\perp}$ F. Olivieri and O. Burastero contributed equally to this work.

\section{Notes}

PDB codes referenced: 4biv, 4biw, 4cb0 (CpxA); 4kp4 (EnvZ $\left.{ }^{\text {chim }}\right)$; 4i5s (VicK); 4u7o (WalK)

The authors declare no competing financial interest.

\section{ABBREVIATIONS}

HK, histidine kinase; DHp, dimerization and histidine phosphoacceptor domain; CA, catalytic and activation domain; $\mathrm{MD}$, molecular dynamics; QM, quantum mechanics; QM/ MM, quantum mechanics/molecular mechanics; DFT, density functional theory; FEP, free energy profile; sMD, steered molecular dynamics; US, umbrella sampling; pHis, phosphoacceptor histidine; HID, $\delta$-protonated; HIE, $\varepsilon$-protonated; HIP, doubly protonated; $\mathrm{DHp}$, dimerization and phosphorylation domain; GGA, generalized gradient approximations; TS, transition state; PBE, Perdue-Burke-Ernzerhof; DZVP, double- $\zeta$ valence polarized

\section{REFERENCES}

(1) Capra, E. J.; Laub, M. T. Evolution of Two-Component Signal Transduction Systems. Annu. Rev. Microbiol. 2012, 66, 325-347.

(2) Koretke, K. K.; Lupas, A. N.; Warren, P. V.; Rosenberg, M.; Brown, J. R. Evolution of Two-Component Signal Transduction. Mol. Biol. Evol. 2000, 17 (12), 1956-1970.

(3) Hoch, J. A.; Silhavy, T. J., Eds.; Two-Component Signal Transduction; American Society for Microbiology, 1995. DOI: $10.1128 / 9781555818319$

(4) Aguilar, P. S.; Hernandez-Arriaga, A. M.; Cybulski, L. E.; Erazo, A. C.; de Mendoza, D. Molecular Basis of Thermosensing: A TwoComponent Signal Transduction Thermometer in Bacillus Subtilis. EMBO J. 2001, 20 (7), 1681-1691.

(5) Albanesi, D.; Martín, M.; Trajtenberg, F.; Mansilla, M. C.; Haouz, A.; Alzari, P. M.; de Mendoza, D.; Buschiazzo, A. Structural Plasticity and Catalysis Regulation of a Thermosensor Histidine Kinase. Proc. Natl. Acad. Sci. U. S. A. 2009, 106 (38), 16185-16190.

(6) Cheung, J.; Hendrickson, W. A. Sensor Domains of TwoComponent Regulatory Systems. Curr. Opin. Microbiol. 2010, 13 (2), 116-123. 
(7) Groisman, E. A. The Pleiotropic Two-Component Regulatory System PhoP-PhoQ. J. Bacteriol. 2001, 183 (6), 1835-1842.

(8) Kaspar, S.; Perozzo, R.; Reinelt, S.; Meyer, M.; Pfister, K.; Scapozza, L.; Bott, M. The Periplasmic Domain of the Histidine Autokinase CitA Functions as a Highly Specific Citrate Receptor. Mol. Microbiol. 1999, 33 (4), 858-872.

(9) Miller, S. I.; Kukral, A. M.; Mekalanos, J. J. A Two-Component Regulatory System (phoP phoQ) Controls Salmonella Typhimurium Virulence. Proc. Natl. Acad. Sci. U. S. A. 1989, 86 (13), 5054-5058.

(10) Boon, C.; Dick, T. Mycobacterium Bovis BCG Response Regulator Essential for Hypoxic Dormancy. J. Bacteriol. 2002, 184 (24), 6760-6767.

(11) Shoji, M.; Cui, L.; Iizuka, R.; Komoto, A.; Neoh, H.-M.; Watanabe, Y.; Hishinuma, T.; Hiramatsu, K. walK and clpP Mutations Confer Reduced Vancomycin Susceptibility in Staphylococcus Aureus. Antimicrob. Agents Chemother. 2011, 55 (8), 3870-3881.

(12) Hu, J.; Zhang, X.; Liu, X.; Chen, C.; Sun, B. Mechanism of Reduced Vancomycin Susceptibility Conferred by walK Mutation in Community-Acquired Methicillin-Resistant Staphylococcus Aureus Strain MW2. Antimicrob. Agents Chemother. 2015, 59 (2), 1352-1355.

(13) Gardete, S.; Kim, C.; Hartmann, B. M.; Mwangi, M.; Roux, C. M.; Dunman, P. M.; Chambers, H. F.; Tomasz, A. Genetic Pathway in Acquisition and Loss of Vancomycin Resistance in a Methicillin Resistant Staphylococcus Aureus (MRSA) Strain of Clonal Type USA300. PLoS Pathog. 2012, 8, No. e1002505.

(14) Gao, R; Stock, A. M. Biological Insights from Structures of Two-Component Proteins. Annu. Rev. Microbiol. 2009, 63, 133-154.

(15) Bhate, M. P.; Molnar, K. S.; Goulian, M.; DeGrado, W. F. Signal Transduction in Histidine Kinases: Insights from New Structures. Structure 2015, 23 (6), 981-994.

(16) Rinaldi, J.; Fernández, I.; Poth, L. M.; Shepard, W. E.; Savko, M.; Goldbaum, F. A.; Klinke, S. Crystallization and Initial X-Ray Diffraction Analysis of the Multi-Domain Brucella Blue LightActivated Histidine Kinase LOV-HK in Its Illuminated State. Biochemistry and Biophysics Reports. 2018, 16, 39-43.

(17) Jacob-Dubuisson, F.; Mechaly, A.; Betton, J.-M.; Antoine, R. Structural Insights into the Signalling Mechanisms of TwoComponent Systems. Nat. Rev. Microbiol. 2018, 16 (10), 585-593.

(18) Shi, T.; Lu, Y.; Liu, X.; Chen, Y.; Jiang, H.; Zhang, J. Mechanism for the Autophosphorylation of CheA Histidine Kinase: QM/MM Calculations. J. Phys. Chem. B 2011, 115 (41), 1189511901 .

(19) Marsico, F.; Burastero, O.; Defelipe, L. A.; Lopez, E. D.; Arrar, M.; Turjanski, A. G.; Marti, M. A. Multiscale Approach to the Activation and Phosphotransfer Mechanism of CpxA Histidine Kinase Reveals a Tight Coupling between Conformational and Chemical Steps. Biochem. Biophys. Res. Commun. 2018, 498 (2), 305-312.

(20) Takada, H.; Yoshikawa, H. Essentiality and Function of WalK/ WalR Two-Component System: The Past, Present, and Future of Research. Biosci., Biotechnol., Biochem. 2018, 82, 741.

(21) Casino, P.; Miguel-Romero, L.; Marina, A. 4KP4: Deciphering Cis-Trans Directionality and Visualizing Autophosphorylation in Histidine Kinases. Nat. Commun. 2014, 5, 3258.

(22) Bottaro, S.; Lindorff-Larsen, K. Biophysical Experiments and Biomolecular Simulations: A Perfect Match? Science 2018, 361 (6400), 355-360.

(23) Defelipe, L. A.; Lanzarotti, E.; Gauto, D.; Marti, M. A.; Turjanski, A. G. Protein Topology Determines Cysteine Oxidation Fate: The Case of Sulfenyl Amide Formation among Protein Families. PLoS Comput. Biol. 2015, 11 (3), No. e1004051.

(24) Zeida, A.; Guardia, C. M.; Lichtig, P.; Perissinotti, L. L.; Defelipe, L. A.; Turjanski, A.; Radi, R.; Trujillo, M.; Estrin, D. A. Thiol Redox Biochemistry: Insights from Computer Simulations. Biophys. Rev. 2014, 6 (1), 27-46.

(25) Jarzynski, C. Nonequilibrium Equality for Free Energy Differences. Phys. Rev. Lett. 1997, 78, 2690-2693.

(26) Liphardt, J. Equilibrium Information from Nonequilibrium Measurements in an Experimental Test of Jarzynski's Equality. Science 2002, 296, 1832-1835.
(27) Park, S.; Khalili-Araghi, F.; Tajkhorshid, E.; Schulten, K. Free Energy Calculation from Steered Molecular Dynamics Simulations Using Jarzynski's Equality. J. Chem. Phys. 2003, 119, 3559-3566.

(28) Leach. Molecular Modelling: Principles And Applications, 2/E; Pearson Education India, 2009.

(29) Fabret, C.; Hoch, J. A. A Two-Component Signal Transduction System Essential for Growth of Bacillus Subtilis: Implications for AntiInfective Therapy. J. Bacteriol. 1998, 180 (23), 6375-6383.

(30) Fukuchi, K.; Kasahara, Y.; Asai, K.; Kobayashi, K.; Moriya, S.; Ogasawara, N. The Essential Two-Component Regulatory System Encoded by yycF and yycG Modulates Expression of the ftsAZ Operon in Bacillus Subtilis. Microbiology 2000, 146 (7), 1573-1583.

(31) Wagner, C.; Saizieu, Ad A. de; Schönfeld, H.-J.; Kamber, M.; Lange, R.; Thompson, C. J.; Page, M. G. Genetic Analysis and Functional Characterization of the Streptococcus Pneumoniae Vic Operon. Infect. Immun. 2002, 70 (11), 6121-6128.

(32) Dubrac, S.; Bisicchia, P.; Devine, K. M.; Msadek, T. A Matter of Life and Death: Cell Wall Homeostasis and the WalKR (YycGF) Essential Signal Transduction Pathway. Mol. Microbiol. 2008, 70 (6), $1307-1322$

(33) Wang, C.; Sang, J.; Wang, J.; Su, M.; Downey, J. S.; Wu, Q.; Wang, S.; Cai, Y.; Xu, X.; Wu, J.; et al. Mechanistic Insights Revealed by the Crystal Structure of a Histidine Kinase with Signal Transducer and Sensor Domains. PLoS Biol. 2013, 11 (2), No. e1001493.

(34) Šali, A.; Blundell, T. L. Comparative Protein Modelling by Satisfaction of Spatial Restraints. J. Mol. Biol. 1993, 234, 779-815.

(35) HMMER: biosequence analysis using profile hidden Markov models. http://hmmer.org (accessed September 13, 2019).

(36) Case, D. A.; Ben-Shalom, I. Y.; Brozell, S. R.; Cerutti, D. S.; Cheatham, T. E., III; Cruzeiro, V. W. D.; Darden, T. A.; Duke, R. E.; Ghoreishi, D.; Gilson, M. K.; Gohlke, H.; Goetz, A. W.; Greene, D.; Harris, R.; Homeyer, N.; Izadi, S.; Kovalenko, A.; Kurtzman, T.; Lee, T. S.; LeGrand, S.; Li, P.; Lin, C.; Liu, J.; Luchko, T.; Luo, R.; Mermelstein, D. J.; Merz, K. M.; Miao, Y.; Monard, G.; Nguyen, C.; Nguyen, H.; Omelyan, I.; Onufriev, A.; Pan, F.; Qi, R.; Roe, D. R.; Roitberg, A.; Sagui, C.; Schott-Verdugo, S.; Shen, J.; Simmerling, C. L.; Smith, J.; Salomon-Ferrer, R.; Swails, J.; Walker, R. C.; Wang, J.; Wei, H.; Wolf, R. M.; Wu, X.; Xiao, L.; York, D. M.; Kollman, P. A. AMBER 2018; University of California: San Francisco, 2018.

(37) Maier, J. A.; Martinez, C.; Kasavajhala, K.; Wickstrom, L.; Hauser, K. E.; Simmerling, C. ff14SB: Improving the Accuracy of Protein Side Chain and Backbone Parameters from ff99SB. J. Chem. Theory Comput. 2015, 11, 3696-3713.

(38) Price, D. J.; Brooks, C. L., 3rd. A Modified TIP3P Water Potential for Simulation with Ewald Summation. J. Chem. Phys. 2004, 121 (20), 10096-10103.

(39) Meagher, K. L.; Redman, L. T.; Carlson, H. A. Development of Polyphosphate Parameters for Use with the AMBER Force Field. J. Comput. Chem. 2003, 24 (9), 1016-1025.

(40) Grossfield, A. WHAM: the weighted histogram analysis method. http://membrane.urmc.rochester.edu/wordpress/?page_id= 126 (accessed September 13, 2019).

(41) Mongan, J.; Case, D. A.; McCammon, J. A. Constant pH Molecular Dynamics in Generalized Born Implicit Solvent. J. Comput. Chem. 2004, 25 (16), 2038-2048.

(42) Nitsche, M. A.; Ferreria, M.; Mocskos, E. E.; González Lebrero, M. C. GPU Accelerated Implementation of Density Functional Theory for Hybrid QM/MM Simulations. J. Chem. Theory Comput. 2014, 10 (3), 959-967.

(43) LIO Project. https://github.com/MALBECC/lio (accessed January 2020).

(44) Perdew, J. P.; Burke, K.; Ernzerhof, M. Generalized Gradient Approximation Made Simple. Phys. Rev. Lett. 1996, 77, 3865; Phys. Rev. Lett. 1997, 78, 1396-1396.

(45) Ramírez, C. L.; Martí, M. A.; Roitberg, A. E. Steered Molecular Dynamics Methods Applied to Enzyme Mechanism and Energetics. Methods Enzymol. 2016, 578, 123-143.

(46) Turjanski, A. G.; Hummer, G.; Gutkind, J. S. How MitogenActivated Protein Kinases Recognize and Phosphorylate Their 
Targets: A QM/MM Study. J. Am. Chem. Soc. 2009, 131 (17), 61416148.

(47) Marina, A.; Mott, C.; Auyzenberg, A.; Hendrickson, W. A.; Waldburger, C. D. Structural and Mutational Analysis of the PhoQ Histidine Kinase Catalytic Domain. Insight into the Reaction Mechanism. J. Biol. Chem. 2001, 276 (44), 41182-41190.

(48) Liepinsh, E.; Otting, G. Proton Exchange Rates from Amino Acid Side Chains- Implications for Image Contrast. Magn. Reson. Med. 1996, 35, 30-42.

(49) Ashenberg, O.; Keating, A. E.; Laub, M. T. Helix Bundle Loops Determine Whether Histidine Kinases Autophosphorylate in Cis or in Trans. J. Mol. Biol. 2013, 425, 1198-1209. 